Z Rheumatol 2021 · 80:936-942

https://doi.org/10.1007/s00393-021-01098-w

Angenommen: 9. September 2021

Online publiziert: 7. Oktober 2021

(๑) Der/die Autor(en) 2021

Redaktion

Axel Hueber, Erlangen

Jutta Richter, Düsseldorf

\section{( )}

\section{In diesem Beitrag}

- Patient reported outcomes

- Elektronisch erfasste „patient reported outcomes" und Datenerhebung in der Rheumatologie

- Studien zur Telemedizin in der Rheumatologie

„Remote monitoring" - PatientInnenpriorisierung und Diagnostik - Smartphone-Applikationen als Monitoringhilfe

- Zukunftsperspektiven und ethische Überlegungen

\title{
Telemedizinische Betreuung und IT-gestützte Verfahren in der Rheumatologie
}

\author{
Rick McCutchan' · Philipp Bosch ${ }^{2}$ \\ ' Universitätsklinik für Innere Medizin II, Medizinische Universität Innsbruck, Innsbruck, Österreich \\ ${ }^{2}$ Klinische Abteilung für Rheumatologie und Immunologie, Medizinische Universität Graz, Graz, Österreich
}

\section{Zusammenfassung}

Hintergrund: Die COVID-19-Pandemie, aber auch die immer größere werdende Beanspruchung des Gesundheitssystems führen dazu, dass die Weiterentwicklung von telemedizinischen Angeboten auch in der Rheumatologie in den Vordergrund gerückt ist.

Fragestellung: Welche Evidenz existiert zu telemedizinischen Angeboten in der Rheumatologie?

Material und Methode: Es erfolgt ein narrativer Review zu bestehenden Arbeiten über Telemedizin in der Rheumatologie.

Ergebnisse: Elektronische „patient reported outcomes“ (ePROs) können von PatientInnen von zu Hause aus bestimmt und dem betreuenden Rheumatologen elektronisch geschickt werden. ePROs könnten in Zukunft dabei helfen zu entscheiden, wann eine klinische Visite notwendig ist. Telemedizinische Visiten wurden bereits durchgeführt bei gut eingestellten PatientInnen mit rheumatischen Erkrankungen mit guten Ergebnissen hinsichtlich Sicherheit und Krankheitsverlauf im Vergleich zu konventionellen Face-to-face-Visiten. Telemedizinische Visiten stellen ein interessantes Tool für Terminpriorisierung und Triage dar, wobei automatisierte, algorithmusbasierte Applikationen derzeit für die klinische Routine noch zu ungenau sind. Die Rolle von Smartphone-Applikationen in der Betreuung von PatientInnen mit rheumatischen Erkrankungen ist noch unklar.

Diskussion: Telemedizin stellt eine interessante Option für bestimmte PatientInnengruppen mit rheumatischen Erkrankungen dar. Abgesehen von Forschung an Effektivität und Sicherheit telemedizinischer Maßnahmen, müssen Entscheidungsträger klare Regeln vorgeben, wie Telemedizin eingesetzt werden soll, um dem individuellen Patienten die bestmögliche Behandlung zukommen zu lassen.

\section{Schlüsselwörter}

Krankheitsaktivität · Digitale Gesundheitsanwendungen · COVID-19 · Patient reported outcomes Algorithmusbasierte Applikationen

Die Begriffe „Telemedizin“ und „remote care" haben in den letzten Monaten aufgrund der COVID-19-Pandemie einen neuen Stellenwert in der Medizin erhalten. Ein deutlicher Anstieg an Terminverschiebungen und Verzögerungen therapeutischer Maßnahmen, die v. a. während der ersten COVID-19-Infektionswelle auftraten, führten dazu, dass eine adäquate PatientInnenversorgung größtenteils nicht mehr möglich war [5]. Insbesondere im Bereich der Rheumato- logie, einem Fach, in dem frühe Diagnosen und Therapien maßgeblich für den Therapieerfolg sind, scheint die Etablierung und Weiterentwicklung telemedizinischer Systeme daher essenziell.

Telemedizin befasst sich mit der Verwendung von Telekommunikation und virtueller Technologie zur Überbrückung von Distanzen bei der Patientlnnenbetreuung. Sie bietet hierbei eine Reihe an Möglichkeiten, angefangen von Terminpriorisierung 
für PatientInnen mit vermeintlich rheumatischen Erkrankungen über diagnostische Erstgespräche bis hin zu kurz- und langfristigen Monitoringvisiten.

Die Nutzung von telemedizinischen Angeboten ist hierbei nicht nur auf Pandemiezeiten beschränkt, sondern bietet auch - neben positiven finanziellen Aspekten die Möglichkeit einer weitreichenden Versorgung in Gebieten mit geringem Angebot an rheumatologischen Ambulanzen [17]. Die Erfassung und Speicherung von PatientInnendaten auf digitale Art und Weise (über Websites, Smartphone-Apps etc.) hat außerdem den Vorteil der einfachen Schaffung von Registerdatenbanken, mithilfe derer Forschung mit dem Ziel der verbesserten PatientInnenversorgung betrieben werden kann.

Um die telemedizinische PatientInnenversorgung mit dem Goldstandard der F2F(, face-to-face")-Betreuung zu vergleichen, gilt es zuerst zu verstehen, welche Daten telemedizinisch erhoben werden können.

\section{Patient reported outcomes}

Um die Krankheitsaktivität nicht nur anhand von radiologischen Gelenkveränderungen, geschwollenen und schmerzhaften Gelenken, sondern auch anhand der subjektiven Einschätzung der PatientInnen bewerten zu können, wurden bereits in den 1970er-Jahren die ersten „patient reported outcomes" (ePROs) entwickelt. Das Ziel dieser war es, die Gesamtheit der Faktoren zu evaluieren, die den Leidensdruck und die alltägliche Funktion bei Menschen mit einer chronischen Erkrankung ausmachen, und durch ihre Erfassung bessere Therapieziele zu formulieren. Zwei der ersten PROs waren der Health Assessment Questionnaire (HAQ) [7] sowie die Arthritis Impact Measurement Scale (AIMS) [19]. Der $\mathrm{HAQ}$ versucht das alltägliche Leben mithilfe von Angaben zur Fähigkeit, sich anzuziehen, aufzustehen, zu essen, Körperpflege zu betreiben etc., zu erfassen. Mobilität, soziale Funktion und Aktivität, körperliche und Alltagsaktivität, Schmerzen, Depressionen, Ängste und Geschicklichkeit sind die 9 Hauptgruppen der AIMS, deren Fragenpool aus insgesamt 46 Fragen besteht.
Nach einigen Modifikationen und Erneuerungen dieser Tests haben sich das Feld der PROs und die Anzahl der Messmöglichkeiten rasant erweitert. In einem Review von 2011 waren es 250 verschiedene Messmöglichkeiten [14], wobei die European Alliance of Associations for Rheumatology (EULAR) sogar eine eigene Website betreut, die die verschiedenen Outcome-Parameter in multiplen Sprachen auflistet und aktualisiert [11].

Durch die fortschreitende Digitalisierung werden PRO-Papierfragebögen kontinuierlich von elektronisch erfassten „patient reported outcomes" (ePROs) abgelöst. Vermeidung von Übertragungsfehlern, verringerte administrative Tätigkeiten und damit verbundene Kosteneinsparungen sind nur einige potenzielle Vorteile von ePROs [3]. Studien zeigten des Weiteren, dass die elektronische Erfassung von PROs die gleichen Ergebnisse bringt wie die herkömmliche Erfassung mittels $\mathrm{Pa}$ pierfragebögen [35].

\section{I) Durch die fortschreitende Digitalisierung werden PRO- Papierfragebögen von ePROs abgelöst}

Während PRO-Papierfragebögen in erster Linie direkt in den Ambulanzen und Warteräumen („on-site ${ }^{\prime \prime}$ ) ausgefüllt werden, ermöglichen ePROs die Datenerfassung und Datensendung aus den eigenen 4 Wänden („off-site“). Man könnte sogar argumentieren, dass aufgrund der vertrauten Umgebung die hierbei erhobenen Daten die eigentliche Krankheitsaktivität genauer widerspiegeln als Daten, die in einer medizinischen Ambulanz angegeben werden (ähnlich dem White-Coat-Syndrom bei der arteriellen Hypertonie) [25].

In Bezug auf das Gerät, mit dem die digitale Datenerfassung erfolgt, ist das BYOD(„bring your own device")-Konzept auf dem Vormarsch. Der konventionelle Weg war bisher, den PatientInnen ein Gerät im Rahmen der Datenerhebung (meist in den Kliniken, also „on-site“) zur Verfügung zu stellen. Diese Herangehensweise ist mit einigen Schwachpunkten vergesellschaftet, die Kosten, Versorgungsangebot, Einarbeitung des betreuenden Personals und die Erhaltung des Geräts beinhalten. Aufgrund der nun alltäglichen und breiten Nutzung von Computern und Smartphones ist daher anzunehmen, dass die Datenerfassung in Zukunft immer mehr auf den eigenen elektronischen Geräten erfolgen wird [3].

\section{Elektronisch erfasste „patient reported outcomes" und Datenerhebung in der Rheumatologie}

(e)PROs wie der "Routine Assessment of Patient Index Data 3"(RAPID-3)- oder der "Rheumatoid Arthritis Impact of Disease"(RAID)-Fragebogen werden bereits seit Längerem in der klinischen Routine verwendet, um sich eine bessere Vorstellung von dem subjektiven Krankheitserleben der individuellen PatientInnen machen zu können [10, 24]. Diese (e)PROs können von PatientInnen prinzipiell problemlos digital ausgefüllt und den betreuenden ÄrztInnen vor einer Visite zugeschickt werden [31].

Ähnliches gilt für Laborparameter, die im Vorhinein bei HausärztInnen oder bei einem Institut abgenommen werden können. Schwieriger wird es bei der körperlichen Untersuchung. Während Gelenkschmerzen von Patientlnnen recht gut lokalisiert werden können, insbesondere wenn sie sich in einem Zustand der geringen Krankheitsaktivität befinden, ist die Einschätzung einer Gelenkschwellungen oftmals unterschiedlich von der von RheumatologInnen [2, 27]. Die British Society of Rheumatology hat nichtsdestotrotz ein Video veröffentlicht, in dem PatientInnen beigebracht wird, einen Gelenkstatus selbst durchzuführen [13] mit dem Ziel der Sammlung dieser Informationen über eine Smartphone-App in eine elektronische Krankenakte (- Abb. 1; [1]).

PatientInnen, die nach einer Schulung in der Lage sind, einen adäquaten Gelenkstatus durchzuführen, wären ideale KandidatInnen für telemedizinische Visiten. Krankheitsaktivitätsscores wie der "Clinical Disease Activity Index" (cDAl) könnten so berechnet und den RheumatologInnen im Vorhinein zur Verfügung gestellt werden. Die alleinige Verwendung von ePROs hingegen könnte in Zukunft den Nutzen bringen zu evaluieren, ob eine F2F-Visite überhaupt 


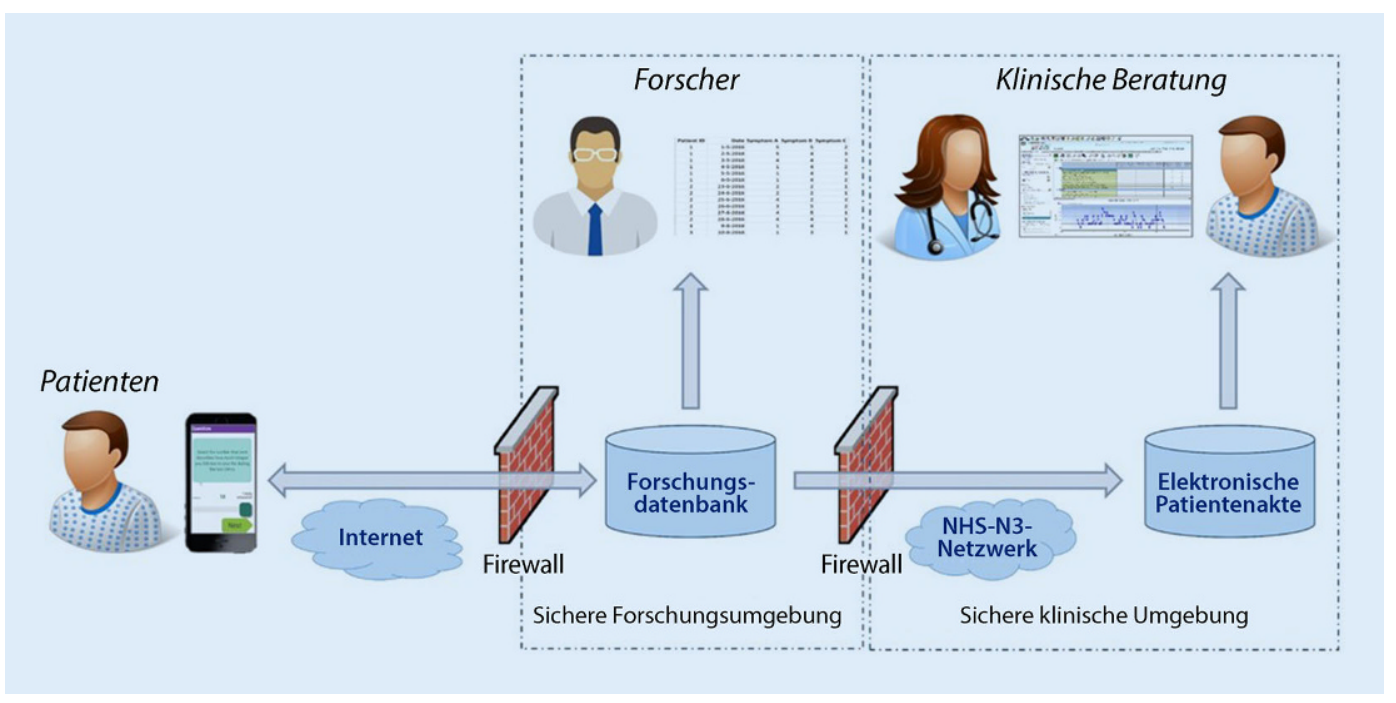

Abb. $1 \varangle$ Datenspeicherung von Smartphone zur elektronischen Krankenakte. (Aus [1]; mit freundl. Genehmigung $\odot$ L. Austin et al., CC BY 4.0 [http:// creativecommons.org/ licenses/by/4.0/]) notwendig ist. Größere Studien hierzu werden jedoch noch benötigt.

Die Bereitschaft von PatientInnen, ihre Daten kontinuierlich und konsequent einzugeben, ist durchaus gegeben, vorausgesetzt dieser folgt eine Reaktion des/der betreuenden Rheumatologln, wenn Werte einer höheren Krankheitsaktivität entsprechen [20]. Generell scheint jedoch noch eine Skepsis gegenüber telemedizinischen Maßnahmen zu bestehen, wie eine rezente Umfrage aus Spanien zeigt. Nur 52,7 \% der befragten PatientInnen mit verschiedenen rheumatischen Erkrankungen gaben an, dass eine Telefonvisite nützlich zum Monitoring ihrer Krankheit wäre, wobei auch nur $37,9 \%$ angaben, bereits eine solche Visite gehabt zu haben [18].

\section{Studien zur Telemedizin in der Rheumatologie}

\section{„Remote monitoring"}

Die wohl einfachste Form des "remote monitoring" stellt die Telefonie dar. Über 4 Mrd. Menschen weltweit haben die Möglichkeit, ein Telefon zu benutzen [12], was sich auch darin ausdrückt, dass die Telefonsprechstunde die meistgenutzte Alternative zur F2F-Visite ist [33].

In einer randomisiert kontrollierten Studie (RCT) aus dem Jahr 2018 mit 294 PatientInnen mit rheumatoider Arthritis, konnte gezeigt werden, dass eine telefonische Visite einer F2F-Visite nicht unterlegen ist, wenn man als primären Endpunkt den DAS(Disease Activity
Score) 28 nach 52 Wochen betrachtet. Insgesamt verringerte sich die Anzahl der F2F-Visiten in dieser Studie um das 4-Fache $(1,75$ [Standardabweichung/SD 1,03] Visiten/Jahr vs. 4,15 [SD 1,0] Visiten/Jahr), wobei auch gezeigt werden konnte, dass die telefonische Visite von Krankenpflegepersonal durchgeführt werden konnte ohne Unterschiede im DAS28, Anzahl an Rezidiven oder Anzahl der F2F-Visiten im Vergleich zu Visiten, die von ÄrztInnen geführt wurden [4].

\section{॥) Telefonvisiten können F2F Visiten ergänzen}

Telemedizin ist jedoch nicht zwangsläufig nur für PatientInnen mit gut eingestellter Krankheitsaktivität eine Option. Pers et al. untersuchten den Nutzen einer Smartphone-Applikation bei PatientInnen mit rheumatoider Arthritis (RA) in moderater/ hoher Krankheitsaktivität, bei denen eine Therapie mit einem "disease-modifying anti-rheumatic drug" (DMARD) eingeleitet wurde [23]. In dieser RCT wurde die erste Hälfte der PatientInnen 1-mal wöchentlich per E-Mail darauf hingewiesen, einige ePROs inklusive RAPID-3 und auto-DAS28 in eine Smartphone-App zu notieren [26]. War der RAPID-3>12/30 Punkten oder wurden nach 2 Monaten kaum Daten von PatientInnen eingegeben, wurden diese von einem "clinical case manager" telefonisch kontaktiert, um einen F2F- oder telefonischen Termin mit einer Rheumatologln auszumachen. In der zweiten Gruppe erfolgten routinemä- ßige F2F-Kontrollen nach Einschätzung der betreuenden Rheumatologln. Nach 6 Monaten fanden sich keine Unterschiede zwischen den Gruppen anhand von DAS28, RAPID-3 oder HAQ. In der Smartphone-App-Gruppe war die Anzahl an F2F-Visiten deutlich geringer als in der Vergleichsgruppe $(0,42$ [SD 0,58 ] vs. 1,93 [SD 0,55]; $p<0,05)$, während die Anzahl an Telefonaten deutlich höher war $(2,67$ [SD 2,58] vs. 0,41 [SD 1,19]; $p<0,01$ ).

Gerade in Situationen, in denen Distanz eine entscheidende Rolle spielt, erscheint der Einsatz von Telemedizin sinnvoll. In einer 2017 veröffentlichten RCT konnte gezeigt werden, dass RA-PatientInnen $(n=85)$ aus dem ländlichen Raum, die eine 3-monatliche Telekonferenz mit einer Rheumatologln hatten, ähnliche Ergebnisse bei DAS28-CRP (Disease activity score 28-C-reactive protein), RADAl (Rheumatoid Arthritis Disease Activity Index), $H A Q$, sowie Lebensqualität und Zufriedenheit hatten im Vergleich zu einer Gruppe, die routinemäßige F2F-Visiten hatte. Hierbei erfolgten die klinische Untersuchung sowie die Unterstützung bei der Telekonferenz durch eine/n Physiotherapeutln [32].

Auch eine rezente chinesische Studie konnte zeigen, dass die Integrierung von Telemedizin gemeinsam mit F2F-Visiten eine Optimierung der Versorgung von PatientInnen mit rheumatischen Erkrankungen darstellen könnte [30], wobei derzeit multiple Studien zu dem Thema in Arbeit sind (- Tab. 1). 
Tab. 1 Ausgewählte Studien zur telemedizinischen Versorgung in der Rheumatologie

\begin{tabular}{|c|c|c|}
\hline Name der Studie & Erkrankungen & Studien-ID \\
\hline Comparing Modes of Telehealth Delivery: Phone vs. Video Visits (ASSISt) & Rheumatische Erkrankungen & NCT04616118 \\
\hline $\begin{array}{l}\text { Effectiveness of Tele-rheumatology for Delivering High Quality Rheumatology Care During the } \\
\text { COVID-19 Crisis (EVOLVE) }\end{array}$ & Rheumatische Erkrankungen & NCT04704544 \\
\hline $\begin{array}{l}\text { Telehealth Stretching Exercise Program for Women with Fibromyalgia During the Covid-19 } \\
\text { Pandemic }\end{array}$ & Fibromyalgie & NCT04690400 \\
\hline $\begin{array}{l}\text { Telehealth CBT for Adolescents and Young Adults with Childhood-onset Systemic Lupus Ery- } \\
\text { thematosus (cSLE) }\end{array}$ & CSLE & NCT04335643 \\
\hline $\begin{array}{l}\text { Evaluation of a Non-face to Face Multidisciplinary Health Care Model in a Population with } \\
\text { Rheumatoid Arthritis }\end{array}$ & RA & NCT04768413 \\
\hline E-learning in Patient Education to Patients with Rheumatoid Arthritis (WEB-RA) & RA & NCT04669340 \\
\hline $\begin{array}{l}\text { Pilot Study of the Impact of Giant Cell Arteritis and Its Treatment on the Autonomy of the } \\
\text { Elderly in the First Year of Care (EPACAPA) }\end{array}$ & GCA & NCT03961113 \\
\hline $\begin{array}{l}\text { Impact of a Hybrid Medical Care Model in the Rheumatoid Arthritis Patient-reported-outcomes } \\
\text { Measures: A Non-inferiority Study }\end{array}$ & RA & NCT04558905 \\
\hline The Effects of Tele-Yoga in Ankylosing Spondylitis Patients & axSpA & NCT04803383 \\
\hline Monitoring Spondyloarthritis With SpA-Net (TeleSpA) & SpA & NCT04673825 \\
\hline $\begin{array}{l}\text { Impact of Barriers and Facilitators to Physical Activity in Patients with Inflammatory Arthri- } \\
\text { tis (ImBAIA) }\end{array}$ & Entzündliche Arthritis & NCT04426747 \\
\hline A Novel Mobile App \& Population Management System to Manage Rheumatoid Arthritis Flares & RA & NCT02822521 \\
\hline $\begin{array}{l}\text { A Multi-Center Clinical Trial to Determine the Impact of a Mobile Health Application on Rheu- } \\
\text { matoid Arthritis Shared Decision Making }\end{array}$ & RA & NCT03773471 \\
\hline Multidisciplinary Approach for Treat To Target In Rheumatoid Arthritis (MAPPIT-RA) & RA & NCT02720874 \\
\hline Walk for Rheumatoid Arthritis (WARA Study) & RA & NCT02467205 \\
\hline
\end{tabular}

\section{PatientInnenpriorisierung und Diagnostik}

In rheumatologischen Ambulanzen finden sich nicht selten PatientInnen mit muskuloskeletalen Beschwerden, die in erster Linie eine orthopädische und nicht rheumatologische Grunderkrankungen vorweisen. PatientInnenpriorisierung und Triage erfolgt bereits an vielen Zentren auf unterschiedliche Art und Weise. In der Rheumatologie-Ambulanz der Medizinischen Universität Innsbruck wird beispielsweise der "rheumatology urgency score" (RUS) verwendet, um die Dringlichkeit eines Termins einzuschätzen [8]. Ein dringlicher Fall wird hierbei bei einem Punktescore von $\geq 4$ definiert (- Abb. 2).

Legget et al. veröffentlichten 2001 Ergebnisse einer Kohortenstudie, in der 100 PatientInnen mit dem Verdacht einer rheumatischen Erkrankung eingeschlossen wurden. Es fand eine rein telefonische Kontaktaufnahme zwischen PatientInnen, HausärztInnen (= ÜberweiserInnen) und Rheumatologinnen statt, wobei sich die PatientInnen dabei in der HausärztInnenPraxis aufhielten. Nach einer initialen Anamnese und Befundbesprechung wurde auch eine Videokamera eingeschaltet. Die RheumatologInnen stellten sowohl nach dem Telefongespräch als auch nach der Videotelefonie eine Verdachtsdiagnose. Die PatientInnen hatten schließlich einen F2F-Termin in der rheumatologischen Ambulanz, wo die finale Diagnose gestellt wurde (=Goldstandard). Die diagnostische Genauigkeit nach dem Telefongespräch war $71 \%$, während jene nach der Videotelefonie $97 \%$ ausmachte [16]. Ähnliche Ergebnisse wurden auch aus einer anderen Kohortenstudie berichtet, bei der die diagnostische Genauigkeit nach einer Videokonferenz bei $79 \%$ lag [21].

Wenngleich diese Ergebnisse Erfolg versprechend klingen, fehlen größere Studien, welche die diagnostische Genauigkeit von telemedizinischen Untersuchungen evaluieren. Online-Tools und Smartphone-Applikationen, die nach Eingabe von Symptomen und Befunden dabei helfen, zwischen einer entzündlich rheumatischen Krankheit und einer anderen Ursache für die Beschwerden zu differenzieren, sind derzeit noch in Erprobung/ Entwicklung bzw. zu ungenau für eine routinemäßige Verwendung in der Praxis [15].
Smartphone-Applikationen als Monitoringhilfe

Da digitale Gesundheitsanwendungen (DiGA) in Deutschland nun in aller Munde sind und die ersten Applikationen zur supportiven Behandlung von PatientInnen mit rheumatischen Erkrankungen schon in den Startlöchern stehen, stellt sich die Frage, inwieweit die Verwendung dieser Programme PatientInnen von Nutzen sein kann.

Eine 2016 veröffentlichte Studie von Salaffi et al. zeigte [29], dass mittels intensiver telemedizinischer Überwachung mehr RA-PatientInnen eine Krankheitsremission ( $\mathrm{CDAl} \leq 2,8)$ nach 1 Jahr erreichten als jene, die routinemäßige Kontrollen hatten $(38,1 \%$ vs. $25 \%, p<0,01)$. Das Studiendesign dieser RCT umfasste 5 F2F-Visiten in beiden Gruppen und zusätzliche 8 telemedizinische Visiten in der Interventionsgruppe. In diesen telemedizinischen Visiten wurde anhand des RADAl bestimmt, ob die Notwendigkeit einer Therapieadaptierung gegeben war. Beim Ausbleiben einer Besserung wurde die Basistherapie entsprechend einem vorgegebenen Schema adaptiert [34]. In der Kontrollgruppe 
Rheumatology Urgency Evaluation for Registration ( $=1,[=2$ )

Please fill out completely!

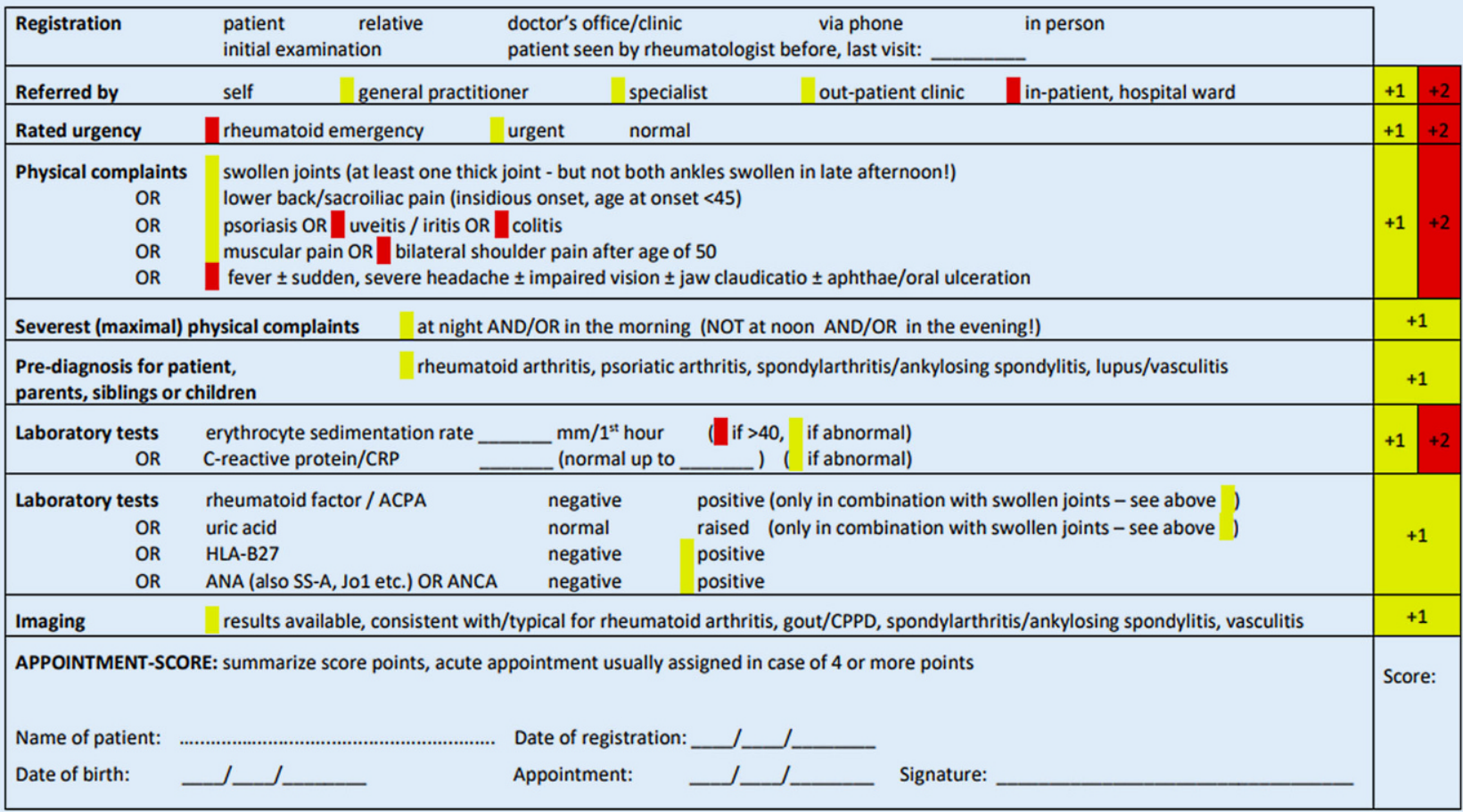

(c) M. Schirmer

Abb. $2 \Delta$ Der "rheumatology urgency score" zur Terminpriorisierung in der rheumatologischen Ambulanz. (Aus [8]; mit freundl. Genehmigung $\odot$ M. Schirmer, alle Rechte vorbehalten)

erfolgte die Therapieadaptierung individuell nach Einschätzung der betreuenden RheumatologInnen.

Eine weitere Gruppe der SmartphoneApplikationen befasst sich mit Symptomtracking: Schrittzähler und Aktivitätsuhren haben nicht nur das Potenzial, durch Motivation die physische Aktivität zu steigern [28], sondern könnten laut einer laufenden RCT in Zukunft auch dabei helfen, Rückschlüsse auf die Krankheitsaktivität zu ziehen. Durch maschinelles Lernen von Daten aus Schrittzählern konnte mit hoher Sensitivität (95,7\%) und Spezifität (96,7\%) festgestellt werden, ob bei PatientInnen mit RA oder Spondyloarthritis ein Krankheitsschub vorlag oder nicht [9]. Ähnliche Studien fanden auch Zusammenhänge zwischen Krankheitsaktivität bei RAPatientInnen (DAS28) und unüblichen Outcomes wie der Händegreiffunktion (gemessen mittels handyverbundenem Dynamometer) [6] sowie der Beschleunigung beim Gehen [22].

\section{Zukunftsperspektiven und ethische Überlegungen}

Die COVID-19-Pandemie hat den Startschuss für den Aufbau und die Weiterentwicklung der telemedizinischen Betreuung in der Rheumatologie gegeben. Laufende Studien zu dem Thema finden sich in Hülle und Fülle (s. - Tab. 1) und befassen sich unter anderem mit der Videotelefonie als Alternative zur F2F-Visite, als Option zur PatientInnenschulung und Anleitung von körperlichen Übungen sowie als Plattform für kognitive Verhaltenstherapie. Außerdem werden nun nach und nach Smartphone-Applikationen und Programme für Patientinnen mit rheumatischen Krankheiten präsentiert, die das Ziel verfolgen, durch Eingabe von Symptomen und subjektiven Krankheitszuständen die PatientInnen in die eigene Behandlung besser einzubinden und die Möglichkeit einer telemedizinischen Betreuung zu erleichtern. Gerade im Hinblick auf die Mög- lichkeit, DiGA zu verschreiben und zu verrechnen, kann man annehmen, dass in den nächsten Jahren eine Vielzahl neuer Gesundheitsapplikationen auf den Markt kommen wird.

\section{I) Telemedizin sollte als} zusätzliche Option und nicht als alleinige Behandlungsmaßnahme verstanden werden

Wie die Telemedizin die PatientInnenbetreuung in der Rheumatologie in Zukunft beeinflussen oder gar revolutionieren wird, ist noch unklar. Ein EULAR-Projekt, das zu diesem Thema "points to consider" entwickelt, ist derzeit in Arbeit und wurde bereits beim EULAR Meeting im Juni 2021 präsentiert. Hier wurden auch die Herausforderungen und ethischen Überlegungen einer telemedizinischen Betreuung diskutiert. Telemedizin sollte für und mit PatientInnen mit rheumatischen Erkrankungen entwickelt werden, wobei im Vordergrund 
die Verbesserung und Erleichterung der PatientInnenbetreuung stehen muss. Trotz Ausbaus telemedizinischer Maßnahmen muss für die PatientInnen weiterhin die Möglichkeit einer konventionellen F2FVisite auf Wunsch möglich sein. Telemedizin sollte daher als zusätzliche Option und nicht als alleinige Behandlungsmaßnahme verstanden werden. Der adäquate Umgang mit PatientInnendaten steht dabei ebenso im Fokus wie die individualisierte Betreuung von PatientInnen mit rheumatischen Erkrankungen.

\section{Fazit für die Praxis}

- Elektronische „patient reported outcomes" (ePROs) bieten langfristige Vorteile im Vergleich zu PROs, die über Papierfragebögen abgefragt werden.

- ePROs haben das Potenzial festzustellen, ob eine klinische Visite bei PatientInnen mit rheumatischen Erkrankungen notwendig ist oder nicht. Größere Studien hierzu fehlen jedoch noch.

- Telemedizinische Visiten wurden zum Monitoring von PatientInnen mit rheumatischen Erkrankungen bereits verwendet, und erste Studien zeigen gute Ergebnisse im Hinblick auf Sicherheit und Krankheitsverlauf.

- Telemedizinische Visiten stellen ein interessantes Tool für Terminpriorisierung und Triage dar, wobei automatisierte, algorithmusbasierte Applikationen derzeit für die klinische Routine noch zu ungenau sind.

- Trotz erster Erfolg versprechender Ergebnisse ist der Wert von Smartphone-Applikationen für die Behandlung und das Monitoring von PatientInnen mit rheumatischen Erkrankungen noch unklar.

- Die Option einer telemedizinischen Visite darf PatientInnen nicht die Möglichkeit nehmen, eine konventionelle Face-toface-Visite in Anspruch zu nehmen.

\section{Korrespondenzadresse}

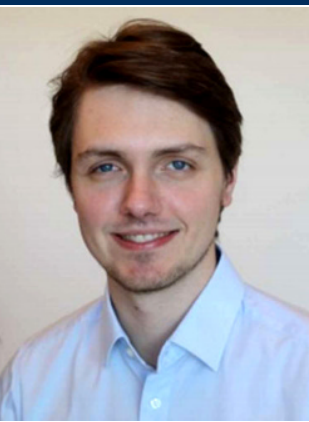

Dr. med. univ. Philipp Bosch

Klinische Abteilung für Rheumatologie und Immunologie, Medizinische Universität Graz Auenbruggerplatz 15, 8036 Graz, Österreich philipp.bosch@medunigraz.at

Funding. Open access funding provided by Medical University of Graz.

\section{Einhaltung ethischer Richtlinien}

Interessenkonflikt. R. McCutchan und P. Bosch geben an, dass kein Interessenkonflikt besteht.

Für diesen Beitrag wurden von den Autoren keine Studien an Menschen oder Tieren durchgeführt. Für die aufgeführten Studien gelten die jeweils dort angegebenen ethischen Richtlinien.

Open Access. Dieser Artikel wird unter der Creative Commons Namensnennung 4.0 International Lizenz veröffentlicht, welche die Nutzung, Vervielfältigung, Bearbeitung, Verbreitung und Wiedergabe in jeglichem Medium und Format erlaubt, sofern Sie den/die ursprünglichen Autor(en) und die Quelle ordnungsgemäß nennen, einen Link zur Creative Commons Lizenz beifügen und angeben, ob Änderungen vorgenommen wurden.

Die in diesem Artikel enthaltenen Bilder und sonstiges Drittmaterial unterliegen ebenfalls der genannten Creative Commons Lizenz, sofern sich aus der Abbildungslegende nichts anderes ergibt. Sofern das betreffende Material nicht unter der genannten Creative Commons Lizenz steht und die betreffende Handlung nicht nach gesetzlichen Vorschriften erlaubt ist, ist für die oben aufgeführten Weiterverwendungen des Materials die Einwilligung des jeweiligen Rechteinhabers einzuholen.

Weitere Details zur Lizenz entnehmen Sie bitte der Lizenzinformation auf http://creativecommons.org/ licenses/by/4.0/deed.de.

\section{Literatur}

1. Austin L, Sharp CA, Van Der VSN et al (2020) Providing "the bigger picture": benefits and feasibility of integrating remote monitoring from smartphones into the electronic health record: findings from the remote monitoring of rheumatoid arthritis (REMORA) study. Baillieres Clin Rheumatol 59:367-378

2. Cheung PP, Ruyssen-Witrand A, Gossec $L$ et al (2010) Reliability of patient self-evaluation of swollen and tender joints in rheumatoid arthritis: a comparison study with ultrasonography, physician, and nurse assessments. Arthritis Care Res 62:1112-1119

3. Coons SJ, Eremenco S, Lundy JJ et al (2015) Capturing patient-reported outcome (PRO) data electronically: the past, present, and promise of ePRO measurement in clinical trials. Patient 8:301-309

4. De Thurah A, Stengaard-Pedersen $K$, Axelsen M et al (2018) Tele-health followup strategy for tight control of disease activity in rheumatoid arthritis: results of a randomized controlled trial. Arthritis Care Res 70:353-360

5. Dejaco C, Alunno A, Bijlsma JW et al (2020) Influence of COVID-19 pandemic on decisions for the management of people with inflammatory rheumatic and musculoskeletal diseases: a survey among EULAR countries. Ann Rheum Dis. https:// doi.org/10.1136/annrheumdis-2020-218697

6. Espinoza F, Le Blay P, Coulon Det al (2016) Handgrip strength measured by a dynamometer connected to a smartphone: a new applied health technology solution for the self-assessment of rheumatoid arthritis disease activity. Baillieres Clin Rheumatol 55:897-901

7. Fries JF, Spitz P, Kraines RGetal (1980) Measurement of patient outcome in arthritis. Arthritis Rheum 23:137-145

8. Ghazal MO, Schirmer M (2017) Proposal for an urgency score as general referral strategy to second-care rheumatology. Ann Musculoskelet Med 1:1-7

9. Gossec L, Guyard F, Leroy D et al (2019) Detection of flares by decrease in physical activity, collected using wearable activity trackers in rheumatoid arthritis or axial spondyloarthritis: an application of machine learning analyses in rheumatology. Arthritis Care Res 71:1336-1343

10. Gossec L, Paternotte S, Aanerud GJ et al (2011) Finalisation and validation of the rheumatoid arthritis impact of disease score, a patient-derived composite measure of impact of rheumatoid arthritis: a EULAR initiative. Ann Rheum Dis 70:935-942

11. http://Oml.Eular.Org/Index.Cfm. Zugegriffen: 9 Juni 2021

12. https://Www.Statista.Com/Statistics/274774/ Forecast-of-Mobile-Phone-Users-Worldwide/. Zugegriffen: 9. Juni 2021

13. https://Www.Youtube.Com/Watch? $V=S b s j k m y n o a w \& T=248 s$. Zugegriffen: 10 . Juni 2021

14. Katz PP (2011) Introduction to special issue: patient outcomes in rheumatology, 2011. Arthritis Care Res 63(Suppl 11):S1-S3

15. Knitza J, Mohn J, Bergmann Cet al (2021) Accuracy, patient-perceived usability, and acceptance of two symptom checkers (Ada and Rheport) in rheumatology: interim results from a randomized controlled crossover trial. Arthritis Res Ther 23:112

16. Leggett P, Graham L, Steele K et al (2001) Telerheumatology-diagnostic accuracy and acceptability to patient, specialist, and general practitioner. Br J Gen Pract 51:746-748

17. Lewtas J (2001) Telemedicine in rheumatology. JRheumatol 28:1745

18. López-Medina C, Escudero A, Collantes-Estevez E (2020) COVID-19 pandemic: an opportunity to assess the utility of telemedicine in patients with 
rheumatic diseases. Ann Rheum Dis. https://doi. org/10.1136/annrheumdis-2020-218070

19. Meenan RF, Gertman PM, Mason JH et al (1982) The arthritis impact measurement scales. further investigations of a health status measure. Arthritis Rheum 25:1048-1053

20. Navarro-Millán I, Zinski A, Shurbaji S et al (2019) Perspectives of rheumatoid arthritis patients on electronic communication and patient-reported outcome data collection: a qualitative study. Arthritis Care Res 71:80-87

21. Nguyen-Oghalai TU, Hunter K, Lyon M (2018) Telerheumatology: the VA experience. South Med J 111:359-362

22. Nishiguchi $\mathrm{S}$, Ito H, Yamada $M$ et al (2014) Selfassessment tool of disease activity of rheumatoid arthritis by using a smartphone application. Telemed JE Health 20:235-240

23. Pers YM, Valsecchi V, Mura T et al (2020) A randomized prospective open-label controlled trial comparing the performance of a connected monitoring interface versus physical routine monitoring in patients with rheumatoid arthritis. Rheumatology. https://doi.org/10.1093/rheumatology/ keaa462

24. Pincus T, Yazici Y, Bergman MJ (2009) RAPID3, an index to assess and monitor patients with rheumatoid arthritis, without formal joint counts: similar results to DAS28 and CDAI in clinical trials and clinical care. Rheum Dis Clin North Am 35:773-778

25. Pioli MR, Ritter AM, De Faria AP et al (2018) White coat syndrome and its variations: differences and clinical impact. Integr Blood Press Control 11:73-79

26. Potter T, Wild A, Edwards K et al (2006) Patients' own ability to assess activity of their rheumatoid arthritis. Baillieres Clin Rheumatol 45:1044-1044

27. Radner H, Grisar J, Smolen JS et al (2012) Value of self-performed joint counts in rheumatoid arthritis patients near remission. Arthritis Res Ther 14:R61

28. Ridgers ND, Timperio A, Brown $\mathrm{H}$ et al (2017) A cluster-randomised controlled trial to promote physical activity in adolescents: the Raising Awareness of Physical Activity (RAW-PA) Study. BMC Public Health 17:6-6

29. Salaffi F, Carotti M, Ciapetti A et al (2016) Effectiveness of a telemonitoring intensive strategy in early rheumatoid arthritis: comparison with the conventional management approach. BMC Musculoskelet Disord 17:146

30. So H, Chow E, Cheng I, Lau X, Li T, Szeto C, Tam L (2021) Use of Telemedicine for Follow-up of Lupus Nephritis in the COVID-19 Outbreak: The 6-month Results of a Randomized Controlled Trial [abstract]. Arthritis Rheumatol 73(suppl 10)

31. Solomon DH, Rudin RS (2020) Digital health technologies: opportunities and challenges in rheumatology. Nat Rev Rheumatol 16:525-535

32. Taylor-Gjevre R, Nair B, Bath B et al (2018) Addressing rural and remote access disparities for patients with inflammatory arthritis through video-conferencing and innovative inter-professional care models. Musculoskelet Care 16:90-95

33. Thwaites C, Ryan S, Hassell A (2008) A survey of rheumatology nurse specialists providing telephone helpline advice within England and Wales. Rheumatology 47:522-525

34. Verstappen SM, Jacobs JW, Van Der Veen MJ et al (2007) Intensive treatment with methotrexate in early rheumatoid arthritis: aiming for remission. Computer Assisted Management in Early Rheumatoid Arthritis (CAMERA, an open-label strategy trial). Ann Rheum Dis 66:1443-1449

\section{Telemedical care and IT-based systems in rheumatology}

Background: The coronavirus disease 2019 (COVID-19) pandemic and also the everincreasing demands on the healthcare system, have led to a focus on the further development of telemedical services in rheumatology.

Objective: What is the evidence for telemedical services in rheumatology?

Material and methods: Narrative review of existing literature on telemedicine in rheumatology.

Results: Electronic patient reported outcomes (ePROs) can be determined by patients from their home and sent electronically to the rheumatologist. In future, ePROs may help with the decision whether a patient needs to attend the clinic for a visit or the visit can be rescheduled due to remission and well-being. Telemedicine has already been used for well-controlled patients with rheumatic diseases with good results in terms of safety and disease activity compared to conventional face-to-face visits. Telemedicine represents an interesting tool for appointment prioritization and triaging, while automated algorithm-based applications are currently too imprecise for routine clinical use. The role of smartphone applications in the care of patients with rheumatic diseases is still unclear.

Discussion: Telemedicine represents an interesting option for certain patient populations with rheumatic diseases. Apart from research on the effectiveness and safety of telemedical interventions, decision makers need to set clear rules on how telemedicine should be used to provide the best possible care for the individual patient.

\section{Keywords}

Disease activity · Digital health applications · Patient reported outcomes · COVID-19 · Algorithmbased applications

35. Zbrozek A, Hebert J, Gogates G et al (2013) Validation of electronic systems to collect patientreported outcome (PRO) data-recommendations for clinical trial teams: report of the ISPOR ePRO systems validation good research practices task force. Value Health 16:480-489 